\title{
Évaluation des pratiques post récolte favorables à la contamination de l'arachide par les mycotoxines dans trois régions de Côte d'Ivoire.
}

\author{
Ama Léthicia Manizana, David Akakib , Isabelle Piro-Metayerc, Didier Montetc, Catherine Brabetc, Rose \\ Koffi-Nevrya \\ a UNA, UFR des Sciences et Technologie des Aliments, Laboratoire de Biotechnologie et Microbiologie des Aliments, 02 \\ BP 801 Abidjan, Côte d'Ivoire rosenevry2002@yahoo.fr; \\ b INP-HB, Département Génie Chimique et Agro-alimentaire, Laboratoire des Procédés Industriels de Synthèse, de \\ I'Environnement et des Énergies Nouvelles, BP 1313 Yamoussoukro, Côte d'Ivoire davidakaki@yahoo.fr \\ c UMR 95 QualiSud / Cirad/Université of Montpellier, TA B-95 /16, 73 rue J-F Breton, 34398 Montpellier cedex 5, France \\ isabelle.piro-metayer@cirad.fr didier.montet@cirad.fr catherine.brabet@cirad.fr; \\ Auteur correspondant: Ama Léthicia Manizan, manizanlethicia@yahoo.fr
}

Original submitted in on $5^{\text {th }}$ February 2018. Published online at www.m.elewa.org on $30^{\text {th }}$ April 2018 https://dx.doi.org/10.4314/jab.v124i1.6

\section{RESUME}

Objectif : Les techniques post récoltes jouent un rôle déterminant dans la contamination de l'arachide par les aflatoxines. Cette étude a pour objectif de contribuer à réduire la contamination de l'arachide par les aflatoxines en Côte d'Ivoire par l'identification des pratiques post récoltes à risque.

Méthodologie et résultats : Un questionnaire a permis de recueillir les renseignements sur lesdites pratiques dans trois régions : nord, centre et ouest. Le séchage de l'arachide se fait au soleil quel que soit la localité et dure en moyenne 4 à 14 jours. Les arachides sont séchées et conservées en coques dans le nord. Dans les zones de centre et ouest, les gousses sont soit séchées puis décortiquées, soit décortiqués avant séchage. Le stockage des graines ou des gousses se fait dans des sacs en polyéthylène dans les maisons ( $86 \%$ ) ou en vrac dans des greniers (14\%). La récolte peut être conservée jusqu'à 9 mois avant consommation ou vente. $58,1 \%$ des productrices ont des pertes dues à l'effet des moisissures. La contamination fongique de l'arachide s'opère dans $55,8 \%$ des cas, durant le séchage et le stockage, et dans $34,9 \%$ des cas au cours de l'apparition des fleurs au champ.

Conclusion et application des résultats : Les étapes de séchage et de stockage représentent un risque de contamination par les aflatoxines. Une maitrise des techniques post récolte permettrait de réduire la contamination par les aflatoxines. II ressort de cette étude qu'une formation des productrices aux bonnes pratiques de production réduirait la contamination parcours aflatoxines.

Mots clés : post-récolte, séchage, conservation, arachide, aflatoxines 
Evaluation of post-harvest practices favorable to the contamination of peanut by mycotoxins in three regions of Côte d'Ivoire.

\section{ABSTRACT}

Objective : Post harvest techniques take a decisive role in peanuts by aflatoxins contamination. The aim of this study is to help to reduce aflatoxin contamination of groundnuts in Côte d'Ivoire by identifying post-harvest practices at risk.

Methodology and results : A survey permit to collect information on post-harvest practices in three regions: north, center and west. Peanuts are dried at sun whatever the locality and lasts on average 4 to 14 days. Peanuts are dried and kept in pods in the north. In the central and western areas, pods are either dried and then shelled, or shelled before drying. The storage of seeds or pods is done in polythene bags in homes (86\%) or bulk in granaries (14\%). Peanuts can be kept until 9 months before consumption or sale. $58.1 \%$ of producers have losses due to effect of molds. Fungal contamination of peanuts occurs in $55.8 \%$ of cases, during drying and storage, and in $34.9 \%$ of cases during flowering in the field.

Conclusion and application of results: Drying and storage stages represent a risk of contamination by aflatoxins. Mastering post-harvest techniques would reduce aflatoxin contamination. This study shows that training producers in good production practices would reduce aflatoxin contamination.

Key words: post-harvest, drying, storage, peanut, aflatoxins

\section{INTRODUCTION}

L'arachide (Arachis hypogaea L.) serait originaire des Antilles, de l'Amérique du Sud (Brésil, Argentine, Paraguay, Bolivie et Pérou) et du Mexique (Hammons, 1994). Aliment de base de plusieurs populations, une consommation bihebdomadaire d'arachide et/ou de ses produits dérivés améliorerait la qualité des régimes alimentaires (Griel et al., 2004). En dépit de sa valeur nutritive, de ses effets positifs sur le traitement de la malnutrition sévère chez l'enfant (Briend, 2001) et de la réduction des risques de maladies cardiovasculaires (Fraser, 1999; Albert et al., 2002), l'arachide peut représenter un risque de santé publique plus ou moins important en fonction des régions. En effet, l'arachide peut être sujette à diverses contaminations par des éléments chimiques toxiques, c'est le cas des mycotoxines. Contaminants naturels de la chaîne alimentaire, les mycotoxines sont des métabolites secondaires produits par des moisissures sur une large gamme de produits agricoles (céréales, arachides, noix, cacao, café, ...). En raison de leurs effets toxiques variés, elles peuvent provoquer des intoxications aiguës ou chroniques chez l'homme et les animaux, parfois mortelles. Parmi les mycotoxines, les aflatoxines produites principalement par des moisissures du genre Aspergillus, sont des contaminants majeurs des arachides. Le sol sert de réservoir pour l'inoculum primaire de $A$. flavus et $A$. parasiticus. Les gousses d'arachide sont ainsi en contact direct avec les champignons aflatoxinogènes (Torres et al., 2014). De plus, l'arachide est l'une des cultures hôtes les plus sensibles à Aspergillus flavus (Diao et al., 2014). Plusieurs études ont révélé la contamination de l'arachide par les aflatoxines au Bénin, au Brésil, en Côte d'Ivoire et au Nigéria ( Hell et al., 2000; Sangare-Tigori et al., 2006 ; Nakai et al., 2008; Ezekiel et al., 2013; Kouadio et al., 2014). La contamination de l'arachide par les aflatoxines peut se produire avant, pendant ou après la récolte, et est liée à de nombreux facteurs. Les facteurs pré-récolte comprennent entre autres, la variété de l'arachide, le type de sol, les espèces de moisissures présentes, les conditions climatiques, les pratiques agricoles, l'activité de l'eau et la maturité de l'arachide. Le moment de la récolte est le principal facteur pendant la récolte. Les facteurs post-récolte sont le séchage, les conditions de stockage et le transport (Cotty et Jaime-Garcia, 2007). De bonnes pratiques postrécolte et de stockage peuvent réduire considérablement la contamination par les aflatoxines (Riley et Norred, 1999). Le séchage est l'étape la plus critique qui affecte la qualité des gousses d'arachides (Anonyme, 2010). Les gousses ayant une teneur en eau voisine de 30 à $40 \%$ à la récolte (Ntare et al., 2008), le séchage permet de réduire la teneur en eau et de ce fait augmenter la 

l'arachide par les mycotoxines dans trois régions de Côte d'Ivoire.

durée de conservation. Le retrait des graines endommagées, le stockage dans des endroits propres et secs et le stockage sur des palettes permettent à l'air de circuler à travers l'arachide réduisant ainsi le développement des moisissures et la production des aflatoxines. La prévention pré ou post récolte de la contamination et de la prolifération des moisissures est l'une des meilleures stratégies pour minimiser la contamination de l'arachide par

\section{MATERIEL ET METHODES}

Pour collecter des informations sur les pratiques post récoltes, une enquête a été diligentée auprès des producteurs de villages de trois régions de la Côte d'Ivoire : nord, centre et ouest. Pour ce faire, chaque producteur a été entretenu sur la base d'un questionnaire relativement aux techniques de séchage et de stockage, aux pertes liées à la récolte, à l'usage du produit de la récolte et les bonnes pratiques de production et de gestion des graines de la qualité de l'arachide. L'enquête a concerné 43 producteurs dont 28 dans la zone centre, 10 au nord et 5 à l'ouest.

\section{RESULTATS}

Techniques de séchage et d'entreposage: Les techniques de séchage varient selon les zones investiguées. La différence se situe au niveau du temps de séchage (4 à 14 jours) et de la forme sous laquelle l'arachide est conservée (gousses, graines). La Figure 1 montre une différence de séchage en fonction des localités. Dans la localité de Korhogo, il existe deux techniques de séchage de durée variable. Les arachides sont séchées et conservées en coques. Dans la zone de Bouaké, les gousses sont soit séchées puis décortiquées, soit décortiquées avant d'être séchées. L'arachide est conservée ici, sous forme de graines. Dans la localité de Daloa, les gousses sont séchées au champ puis au les aflatoxines (Riley et Norred, 1999). A notre connaissance, il n'existe pas d'étude sur les techniques post récolte de l'arachide en Côte 'Ivoire. Cette étude a pour objectif, de contribuer à la réduction de la contamination par les aflatoxines de l'arachide en Côte d'Ivoire à travers l'identification des pratiques post récolte de l'arachide dans de certaines grandes régions productrices.

Analyses statistiques : Les données collectées ont fait d'abord l'objet de codage puis dépouillées à l'aide $d u$ logiciel IBM SPSS Statistics Version 21. Le test de Khideux de Pearson, les valeurs de Phi de Pearson ont permis de déterminer la corrélation entre les variables (techniques de séchage, de séchage, usage du produit de la récolte appliqués) analysées et les zones investiguées. Il existe une corrélation lorsque le degré de signification du Khi-deux est $<0,05$. La force de cette corrélation est déterminée par la valeur du Phi : si Phi > 0,7 , la corrélation entre les variables analysées est forte, dans le cas contraire elle est faible.

village avant d'être décortiquées puis séchées à nouveau et stockées sous forme de graines (Figure 1). Dans les localités de Bouaké et de Daloa, les conditions de stockage sont les mêmes : les graines sont mises dans des sacs en polyéthylène puis entreposés dans les maisons. Par contre, dans la zone de Korhogo, l'entreposage se fait soit dans les maisons soit dans un grenier en vrac ou dans des sacs en polyéthylène. En général, la durée de stockage varie de 1 mois à 12 mois. Les graines peuvent être entreposées pendant 12 mois à Korhogo, de 8 à 9 mois à Bouaké et de 2 à 3 mois à Daloa (Tableau 1). 


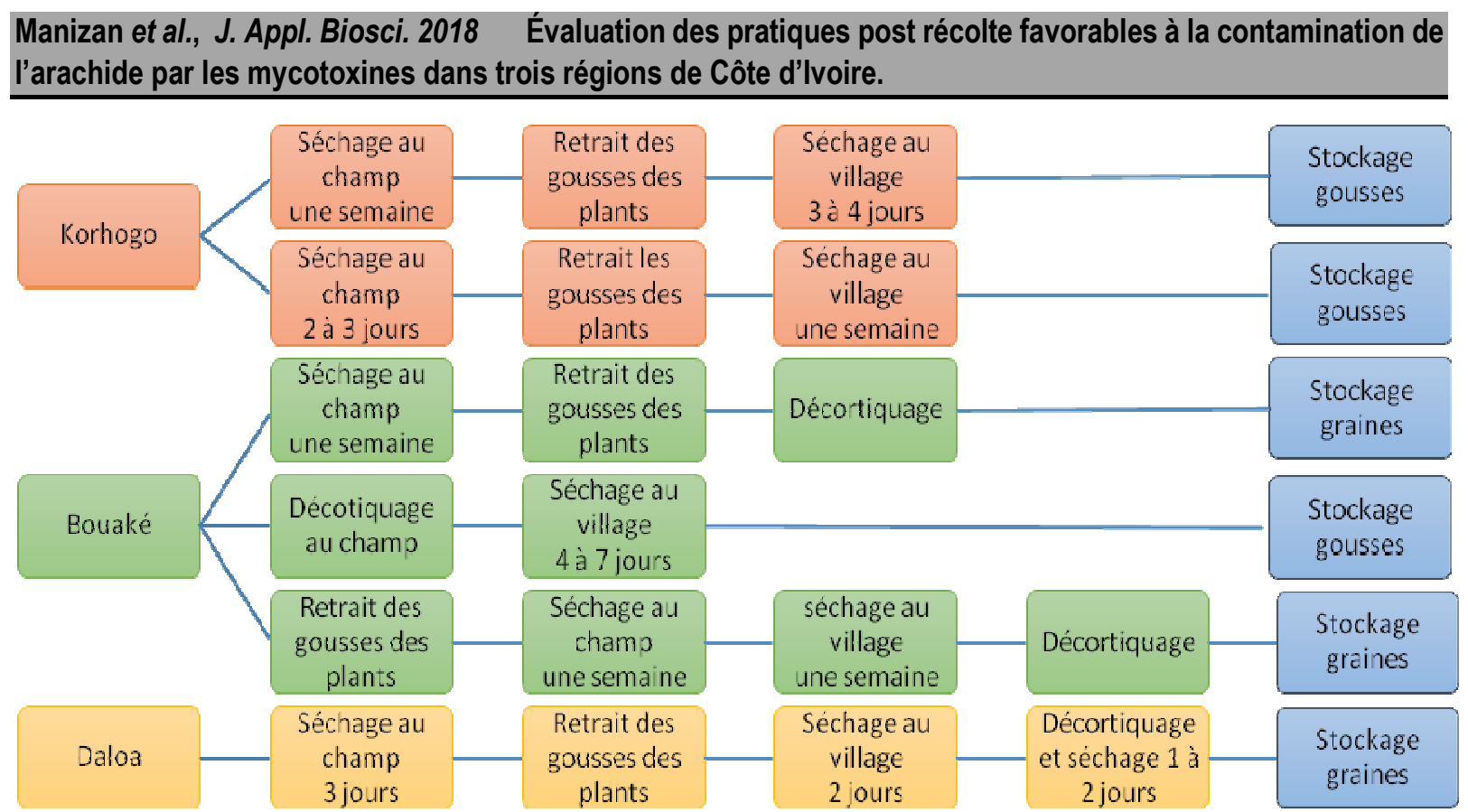

Figure 1 : Techniques de séchage et mode d'entreposage selon les localités

Tableau 1 : Répartition des conditions de stockage des graines d'arachide selon les localités

\begin{tabular}{|c|c|c|c|c|}
\hline \multirow[t]{2}{*}{ Conditions de stockage } & \multicolumn{4}{|c|}{ Pourcentage (n) } \\
\hline & Korhogo $(n=28)$ & Bouaké $(n=10)$ & Daloa $(n=5)$ & $\%$ Total \\
\hline Lieu de stockage (b) & & & & \\
\hline Grenier & $21,4(6)$ & 0 & 0 & 14 \\
\hline Maison & $78,6(22)$ & $100(10)$ & $100(5)$ & 86 \\
\hline Modes de stockage (b) & & & & \\
\hline En sac & $78,6(22)$ & $100(10)$ & $100(5)$ & 86 \\
\hline En vrac & $21,4(6)$ & 0 & 0 & 14 \\
\hline Durée de stockage (a) & & & & \\
\hline 1 an & $100(28)$ & 0 & 0 & 65,1 \\
\hline 8 à 9 mois & 0 & $100(10)$ & 0 & 23,3 \\
\hline 3 mois & 0 & 0 & $40(2)$ & 4,7 \\
\hline 2 mois & 0 & 0 & $60(3)$ & 7 \\
\hline
\end{tabular}

(n) = nombre d'individus ; (a) = forte corrélation entre la variable et la localité (degré de signification du Khi2 $<0,05$ et Phi $>70 \%) ;(b)=$ pas de corrélation entre la variable et la localité (degré de signification du khi2 >0,05).

Pertes liées à la récolte: La majorité, soit $95,3 \%$ des productrices ont affirmé avoir été confrontées à la perte d'une partie de leur récolte (Tableau 2). La perte varie de $2 \%$ à plus de $60 \%$. Les causes sont multiples : les insectes représentent à $81,4 \%$, la principale cause de perte suivis des moisissures $(58,1 \%)$. La cause, la moins fréquente est la germination des graines (7\%). Dans les localités de Korhogo et de Daloa, les productrices sont confrontées aux pertes dues aux insectes et aux moisissures, tandis qu'à Bouaké, en plus des insectes et des moisissures, les pertes sont liées aux maladies des plants et à la germination des graines. À l'exception des productrices de Daloa (100\%) qui utilisent le séchage comme moyen de lutte contre les pertes, aucun moyen de lutte n'est utilisé dans les localités de Korhogo et de Bouaké. La présence de moisissures a été observée au cours du séchage et du stockage dans les zones de Bouaké et Daloa. En plus du séchage et du stockage, l'apparition des moisissures a été observée au cours de la floraison dans la localité de Korhogo. 
Tableau 2 : Répartition des causes de pertes post récolte et moyen de lutte selon les localités

\begin{tabular}{|c|c|c|c|c|}
\hline \multirow[t]{2}{*}{ Pertes post récolte } & \multicolumn{4}{|c|}{ Pourcentage (n) } \\
\hline & Korhogo (n=28) & Bouaké (n=10) & Daloa $(n=5)$ & $\%$ Total \\
\hline $\begin{array}{l}\text { Pertes (b) } \\
\quad 0 \% \\
2-10 \% \\
10-50 \% \\
\geq 60 \%\end{array}$ & $\begin{array}{l}0 \\
53,7(15) \\
42,9(12) \\
3,6(1)\end{array}$ & $\begin{array}{l}20(2) \\
20(2) \\
60(6) \\
0\end{array}$ & $\begin{array}{l}0 \\
80(4) \\
20(1) \\
0\end{array}$ & $\begin{array}{l}4,7 \\
49 \\
44 \\
2,3\end{array}$ \\
\hline \multicolumn{5}{|l|}{ Causes de perte $(a)^{*}$} \\
\hline Insectes & $100(28)$ & $20(2)$ & $100(5)$ & 81,4 \\
\hline Moisissures & $64,3(18)$ & $20(2)$ & $100(5)$ & 58,1 \\
\hline Maladies des plants & 0 & $50(5)$ & 0 & 11,6 \\
\hline Grain germé & 0 & $30(3)$ & 0 & 7 \\
\hline Aucun & 0 & $20(2)$ & 0 & 4,7 \\
\hline \multicolumn{5}{|l|}{ Moyens de lutte (a) } \\
\hline Phytosanitaire & 0 & 0 & 0 & 0 \\
\hline Séchage & & & $100(5)$ & 11,6 \\
\hline Aucun & $100(28)$ & $100(10)$ & & 88,4 \\
\hline \multicolumn{5}{|l|}{ Présence de moisissures (a) } \\
\hline L'apparition des fleurs & $53,6(15)$ & 0 & 0 & 35 \\
\hline Le séchage et le stockage & $46,4(13)$ & $80(8)$ & $80(4)$ & 58 \\
\hline Jamais & 0 & $20(2)$ & $20(1)$ & 7 \\
\hline
\end{tabular}

$(n)=$ nombre d'individus ; (a) = forte corrélation entre la variable et la localité (degré de signification du Khi $2<0,05$ et Phi $>70 \%$ ); (b) = pas de corrélation entre la variable et la localité (degré de signification du khi $2>0,05$ ) ; *a somme des proportions est supérieure à $100 \%$ car un seul individu peut avoir plus d'un choix à cette question.

Usage du produit de la récolte: Le Tableau 3 montre que, quelle que soit la localité, la production d'arachide $(100 \%)$ est soit destinée à l'autoconsommation, soit vendue à des commerçants (grossistes, détaillants) ou aux consommateurs. Dans la localité de Bouaké, toutes les productrices $(100 \%)$ vendent leur récolte sur les marchés directement aux consommateurs. Outre l'autoconsommation, la production d'arachide dans la zone de Korhogo est destinée au commerce avec ou sans intermédiaires. A Daloa, la production est vendue soit à des consommateurs, soit à des revendeurs (commerçants). Les critères de qualité de l'arachide sont basés sur la variété, la teneur en eau (graine bien séchée) et des graines saines (retrait des grains troués, moisis, et de corps étrangers). A l'exception de $50 \%$ des productrices de Korhogo qui ont affirmées avoir eu des refus de leur marchandise suite à la présence de graines moisies, la majorité $(67,4 \%)$ des productrices ont affirmé n'avoir jamais eu de refus de marchandise dû aux moisissures. La présence de moisissure est souvent caractérisée par le changement de couleur des graines (noire, verte, blanche) et la fermeté des graines.

Bonnes pratiques de production et gestion de la qualité de l'arachide: Les bonnes pratiques de production (BPP) sont très peu connues des productrices. En effet, sur l'ensemble des productrices interrogées, seulement $7 \%$ ont reçu une formation sur les bonnes pratiques de production et $14 \%$ ont affirmé les mettre en pratique. Sur l'ensemble des 3 régions soumises à l'étude, il ressort que la contamination fongique de l'arachide s'opère dans $55,8 \%$ des cas, durant le séchage et le stockage, et dans $34,9 \%$ des cas au cours de l'apparition des fleurs au champ (Tableau 4). La technique d'obtention d'arachide de bonne qualité et le stade d'apparition des moisissures sont fonction de la zone. Dans les zones de Korhogo, l'obtention d'arachide de bonne qualité repose sur le tri avant semis $(46,4 \%)$ et le tri pendant le séchage $(46,4 \%)$. A Bouaké, en plus du tri avant le semis ( $50 \%$ ) et au cours du séchage (50\%), $30 \%$ des productrices estiment que la lutte contre les maladies des plants, par l'utilisation de produits phytosanitaires, contribue à une meilleure qualité de l'arachide. Dans la localité de Daloa, les moyens pour obtenir des graines de bonne qualité sont le tri avant semis, pendant la récolte et pendant le séchage respectivement à des pourcentages de $20 \%, 60 \%$ et 20 $\%$. 
Tableau 3 : Distribution de l'usage des récoltes selon les localités

\begin{tabular}{|c|c|c|c|c|}
\hline & \multicolumn{4}{|c|}{ Pourcentage (n) } \\
\hline & Korhogo $(n=28)$ & Bouaké $(n=10)$ & Daloa $(n=5)$ & $\%$ Total \\
\hline \multicolumn{5}{|l|}{ Usage des récoltes (b) } \\
\hline Autoconsommation & 0 & 0 & 0 & 0 \\
\hline Commerce & 0 & 0 & 0 & 0 \\
\hline Autoconsommation et commerce & $100(28)$ & $100(10)$ & $100(5)$ & 100 \\
\hline \multicolumn{5}{|l|}{ Type d'acheteurs $(a)^{*}$} \\
\hline Intermédiaire & $100(28)$ & 0 & 0 & 65,1 \\
\hline Commercant & $53.6(15)$ & 0 & $100(5)$ & 46,5 \\
\hline Consommateur & 0 & $100(100)$ & $60(3)$ & 30,2 \\
\hline \multicolumn{5}{|l|}{ Exigences des acheteurs (a) } \\
\hline Variété & $53,6(15)$ & 0 & 0 & 34,9 \\
\hline Graines non abimées & $39,2(11)$ & $20(2)$ & 0 & 30,3 \\
\hline Graines saines & $7,1(2)$ & 0 & 0 & 4,7 \\
\hline Pas de graines moisies & 0 & $80(8)$ & & 18,6 \\
\hline Aucun & 0 & 0 & $100(5)$ & 11,6 \\
\hline \multicolumn{5}{|l|}{ Refus des graines moisies (c) } \\
\hline Oui & $50(14)$ & 0 & & 32,6 \\
\hline Non & $50(14)$ & $100(10)$ & $100(5)$ & 67,4 \\
\hline
\end{tabular}

(n) = nombre d'individus ; (a) = forte corrélation entre la variable et la localité (degré de signification du Khi2 <0,05 et Phi $>70 \%) ;(b)$ $=$ pas de corrélation entre la variable et la localité (degré de signification du khi2 $>0,05) ;(c)=$ faible de corrélation entre la variable et la localité (degré de signification du khi2 $<0,05$ et Phi $<70 \%$ ). *La somme des proportions est supérieure à $100 \%$ car un seul individu peut avoir plus d'un choix à cette question.

Tableau 4 : Gestion de la qualité de l'arachide en fonction des localités

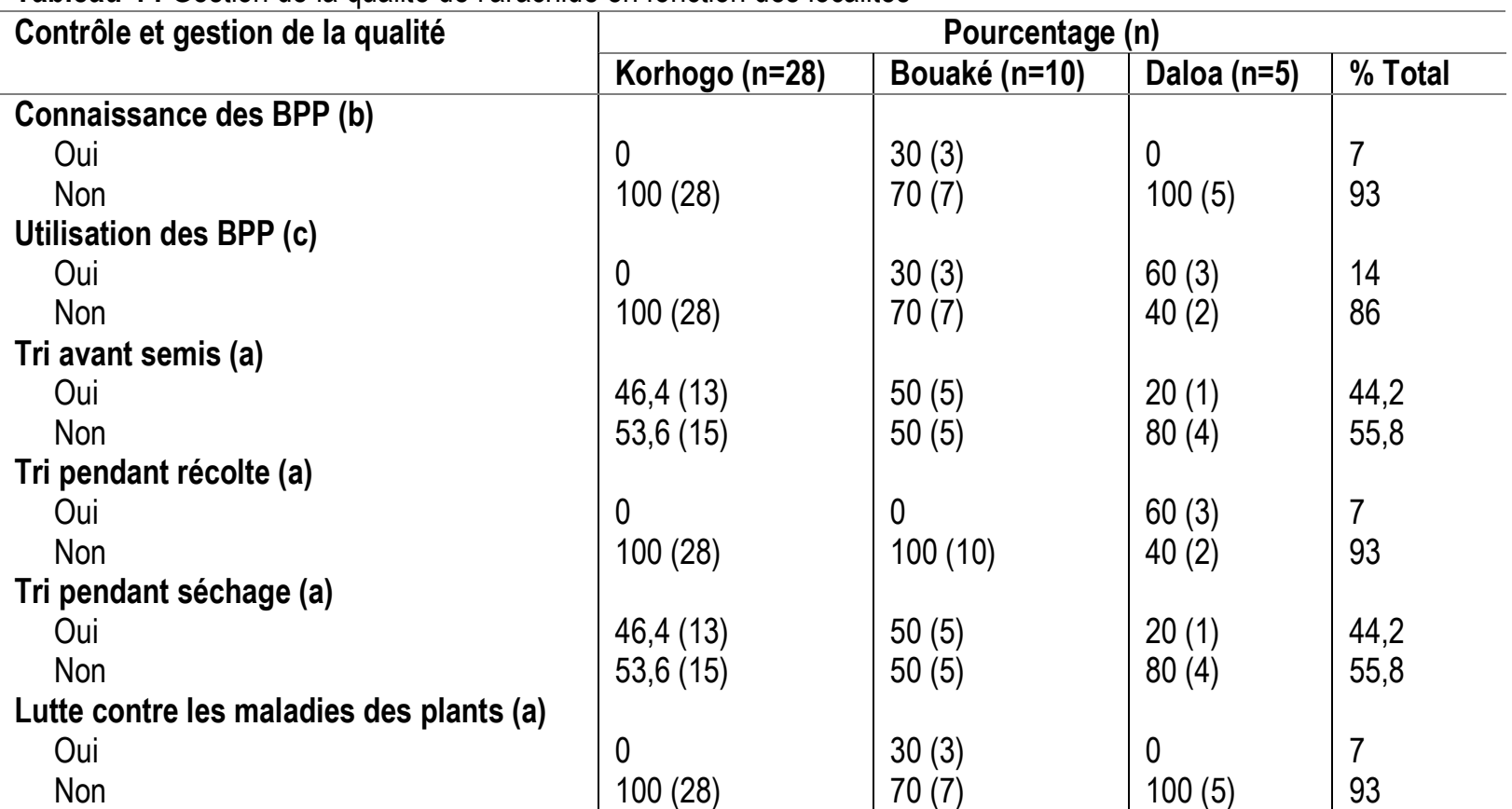

(n) = nombre d'individus ; $(\mathrm{a})=$ forte corrélation entre la variable et la localité (degré de signification du Khi2 <0,05 et Phi $>70 \%) ;(b)$ $=$ pas de corrélation entre la variable et la localité (degré de signification du khi2 $>0,05) ;(c)=$ faible de corrélation entre la variable et la localité (degré de signification du khi2 > 0,05 et Phi > 70\%) 


\section{DISCUSSION}

Les enquêtes réalisées auprès des commerçants ont permis de mettre en évidence les traitements post-récolte de l'arachide pouvant affecter la contamination de l'arachide par les aflatoxines. Les traitements post-récolte à savoir les méthodes, temps de séchage et autres, sont fonction des zones et parfois d'un village à un autre pour une même zone. La durée totale de séchage s'étend de 4 à 14 jours dans les zones soumises à l'étude. Pour ce faire, les gousses sont séchées à même le sol (Korhogo), décortiquées puis séchées sur des sachets (Bouaké) ou séchées puis décortiquées (Bouaké, Daloa). Ces techniques de séchage sont les mêmes que celles rapportées par Demont en 1997, après avoir conduit une étude sur les systèmes de commercialisation des produits vivriers en Côte d'Ivoire. En effet, dans les zones centre et ouest (Bouaké et Daloa), la récolte se fait à la saison pluvieuse. Pour assurer un séchage rapide les productrices égoussent immédiatement et mettent à sécher les graines d'arachide sur des aires de séchage auprès des habitations. Un bon séchage requiert une bonne circulation d'air. L'insuffisance de séchage de l'arachide prédispose à la croissance fongique et à la contamination par les aflatoxines. II est recommandé d'éviter un séchage trop rapide en utilisant des températures très élevées. Cependant, si le séchage dure trop longtemps, la rosée et la lumière du soleil ont tendance à décolorer les graines, tandis que les moisissures peuvent se développer et causer la rupture des gousses (FAO, 2002). Selon Hell et al. (2003), au Benin la contamination post récolte par les aflatoxines a augmentée lorsque la récolte a pris plus de 5 jours et que le séchage a été retarder. Pour une bonne conservation et une bonne germination, le taux d'humidité dans les gousses doit être entre 6 et $8 \%$ (Ntare et al., 2008). Les conditions de conservation des récoltes demeurent les mêmes quelle que soit la zone. Au cours de cette étude, des durées de conservation allant de 2 à 12 mois ont été observées. Les productrices conservent principalement leur récolte dans des sacs en polyéthylène dans leur cuisine ou leur maison ( $86 \%$ ). Quelques-unes (14\%) font un stockage en vrac dans les greniers. Ces résultats sont en cohérence avec ceux de Akoua (2013), qui dans une étude similaire menée au Ghana a montré que $81 \%$ des producteurs stockait leur arachide dans des sacs en polyéthylène. Selon Hell et al. (2000) et aussi Udoh et al. (2000), l'utilisation de sac en polyéthylène de nature peu aéré favorise le développement des moisissures. En effet, le manque d'aération peut être à l'origine d'une variation de la température pouvant entrainer une condensation de l'eau présente dans l'air, augmentant ainsi l'humidité des graines ; ceci risque de favoriser le développement des moisissures. Les locaux de stockage dans les zones visitées sont à usage mixte et très peu aérés. Selon la FAO (2002), la plupart des pays en voie de développement disposent de mauvaises installations de séchage et de stockage. Ces pratiques entrainent des contaminations croisées et la prolifération de moisissures et de ravageurs, augmentant ainsi le risque de contamination par les aflatoxines pendant le stockage. Selon Mutegi et al. (2013), une croissance accrue des moisissures et une production d'aflatoxine serait favorisée par des conditions d'entreposage à haute humidité après récolte. En outre, de longue durée de conservation pourrait aussi favoriser la contamination par les moisissures et la production d'aflatoxines. Un peu moins de la moitié $(46,3 \%)$ des productrices ont affirmé être confrontées à des pertes de plus de $10 \%$ dues principalement aux insectes, aux moisissures et à la germination des graines pendant et après la récolte. Ces pourcentages sont proches de ceux rapportés par Demont (1997). Selon ce dernier, le taux de perte atteint les $15 \%$ de la production totale d'arachide en Côte d'Ivoire. Les dommages causés par les insectes, prédisposent l'arachide à une forte contamination par les moisissures et donc par les mycotoxines. Les ouvertures laissées par les larves de Caryedon serratus par exemple facilitent l'attaque d'autres insectes qui déprécient la qualité nutritive de l'arachide et favorisent le développement de moisissures (Thiaw et al., 2015). La contamination par les moisissures a été signalée par 58 $\%$ des productrices au cours du séchage et du stockage et par $35 \%$ au cours de la floraison. Selon, la majorité des productrices, le principal moyen d'obtenir des graines d'arachide de bonne qualité, consiste à faire des tris avant semis, au cours de la récolte, du séchage, avant la vente ou la consommation. Le tri représente pour certaines productrices une perte au niveau de la production. Aussi, le retrait des matières étrangères et des mauvaises graines représenterait environ $5 \%$ du stock. Selon, Awuah et al. (2009), 5 à $15 \%$ de la production de l'arachide est perdue au cours du tri. Selon les productrices, le séchage après la récolte peut diminuer les pertes de façon considérable. C'est ainsi que dans la zone de Korhogo, le choix d'une variété à cycle long et le suivi des règles normales de date de semis précoce permet de s'inscrire dans une période de récolte favorable au séchage naturel avec quelques jours d'exposition au soleil. En effet, de mauvaises pratiques de récolte, de séchage et de stockage, de même qu'un transport, une commercialisation et une transformation 
inappropriés contribuent au développement des champignons, principalement des champignons de stockage, telles que les moisissures du genre Aspergillus (Degraeve et al., 2016). La production de la totalité des productrices interrogées sert aussi bien pour l'auto consommation que pour le commerce. La Côte d'Ivoire n'est pas autosuffisante pour l'arachide. Ce qui explique qu'elle n'exporte pas l'arachide produite et que toute la production soit utilisée sur place. Ce résultat corrobore

\section{CONCLUSION}

Les pratiques post récoltes à risques de contamination par les moisissures et par les mycotoxines dans la filière arachide en Côte d'Ivoire sont les conditions de séchage (temps, aires de séchage) et de stockage (lieux, contenants). Un mauvais séchage provoque une croissance fongique et la production d'aflatoxines et

\section{RÉFÉRENCES BIBLIOGRAPHIQUES}

Akoua, A.-G. (2013). The Role of Women in Post-Harvest Handling of Peanuts: The Case of Reducing Aflatoxin Along the Supply Chain in Ghana (Alabama: Graduate Faculty of Auburn University).

Albert, C.M., Michael, G., Walter, C.W., et JoAnn, E.M. (2002). Nut Consumption and Decreased Risk of Sudden Cardiac Death in the Physicians' Health Study. Arch. Intern. Med. 162, 1382-1387.

Anonyme (2010). Good agricultural practices for peanut. TAS 4900-2010. National bureau of agricultural commodity and food standards. Ministry of agriculture and cooperatives of Thailand. R. Gaz. 127.

Awuah, R.T., Fialor, S.C., Binns, A.D., Kagochi, J., et Jolly, C.M. (2009). Factors influencing market participants decision to sort groundnuts along the marketing chain in Ghana. Peanut Sci. 36, 68-76.

Boli, Z.A., Zoue, L.T., Alloue-Boraud, W.A.M., Kakou, C.A., et Koffi-Nevry, R. (2014).Proximate composition and mycological characterization of peanut butter sold in retail markets of Abidjan (Côte d'Ivoire). Journal of Applied Biosciences 72 (1): 5822ख? 29.

Briend, A. (2001). Highly nutrient-dense spreads: a new approach to delivering multiple micronutrients to high-risk groups. Br. J. Nutr. 85, S175.

Cotty, P.J., et Jaime-Garcia, R. (2007). Influences of climate on aflatoxin producing fungi and aflatoxin contamination. Int. J. Food Microbiol. 119, 109115. celui de Demont (1997) qui a indiqué qu'en Côte d'ivoire, $15 \%$ de la récolte est destinée à l'autoconsommation, plus de $60 \%$ est vendue et le reste est utilisé pour la semence de la culture suivante. Cette assertion est également soutenue par Sangare-Tigori et al. (2006) et aussi Boli et al. (2013) qui ont rapporté que l'intégralité de la production d'arachide est utilisée au sein du pays.

réduit la qualité des gousses à la commercialisation et à la consommation. Seul le tri est utilisé comme moyen pour prévenir et réduire la contamination par les moisissures. Une formation des productrices aux bonnes pratiques de production leur permettrait d'améliorer leur rendement et la qualité sanitaire de l'arachide produite.

Degraeve, S., Madege, R.R., Audenaert, K., Kamala, A., Ortiz, J., Kimanya, M., Tilisekwa, B., De Meulenaer, B., et Haesaert, G. (2016). Impact of local pre-harvest management practices in maize on the occurrence of Fusarium species and associated mycotoxins in two agroecosystems in Tanzania. Food Control 59, 225233.

Demont, M. (1997). Système de commercialisation des produits vivriers en Côte d'Ivoire étude pour le maïs (Zea mays) et l'arachide (Arachis hypogaea). Doc. Trav. Nº Proj. "Renforcement Études Agro-Économiques A L'IDESSA' IDESSA - KULeuven 79p.

Ezekiel, C.N., Sulyok, M., Babalola, D.A., Warth, B., Ezekiel, V.C., et Krska, R. (2013). Incidence and consumer awareness of toxigenic Aspergillus section Flavi and aflatoxin B1 in peanut cake from Nigeria. Food Control 30, 596-601.

FAO (Food and Agriculture Organisation) (2002). UEMOA - COTE D'IVOIRE: Appui à la mise en oeuvre de la Politique agricole de l'Union en matière de sécurité alimentaire. Programme régional de sécurité alimentaire (FAO). $31 \mathrm{p}$.

Fraser, G.E. (1999). Nut Consumption, Lipids, and Risk of a Coronary Event. 22, 11-15.

Griel, A.E., Eissenstat, B., Juturu, V., Hsieh, G., et KrisEtherton, P.M. (2004). Improved Diet Quality with Peanut Consumption. J. Am. Coll. Nutr. 23, 660-668.

Hammons, R. O. (1994). The origin and history of the groundnut. In The Groundnut Crop: A Scientific 
Basis for Improvement, (Chapman Hall, London, UK), pp. 24-39.

Hell, K., Cardwell, K.F., et Poehling, H.-M. (2003). Distribution of fungal species and aflatoxin contamination in stored maize in four agroecological zones in Benin, West-Africa. Journal of Phytopathology 151, 690-698.

Hell, K., Cardwell, K.F., Setamou, M., et Poehling, H.-M. (2000). The influence of storage practices on aflatoxin contamination in maize in four agroecological zones of Benin, west Africa. J. Stored Prod. Res. 36, 365-382.

Kouadio, J. H., Lattanzio, V. M. T., Ouattara, D., Kouakou, B., et Visconti, A. (2014). Assessment of mycotoxin exposure in Côte D'Ivoire (Ivory Coast) through multi-biomarker analysis and possible correlation with food consumption patterns. Toxicol Int, 21(3), 248-257.

Mutegi, C., Wagacha, M., Kimani, J., Otieno, G., Wanyama, R., Hell, K., et Christie, M.E. (2013). Incidence of aflatoxin in peanuts (Arachis hypogaea Linnaeus) from markets in Western, Nyanza and Nairobi Provinces of Kenya and related market traits. J. Stored Prod. Res. 52, 118-127.

Nakai, V.K., de Oliveira Rocha, L., Gonçalez, E., Fonseca, H., Ortega, E.M.M., et Corrêa, B. (2008). Distribution of fungi and aflatoxins in a stored peanut variety. Food Chem. 106, 285290.

Ntare, B.R., Diallo, A.T., Ndjeunga, J., et Waliyar, F. (2008). Groundnut seed production manual (International Crops Research Institute for the Semi-Arid Tropics).

Riley, R.T., et Norred W.P. (1999). Mycotoxin prevention and decontamination. A case study on maize, in Alimantatio, Nutrition et Agriculture, FAO, 23:2531.

Sangare-Tigori, Dem, A., Kouadio, H., Betbeder, A.-M., Dano, D., Moukha, S., et Creppy, E. (2006). Preliminary survey of ochratoxin A in millet, maize, rice and peanuts in Côte d'Ivoire from 1998 to 2002. Hum. Exp. Toxicol. 25, 211-216.

Thiaw, C., Coly, E., Djiba, S., Diop, M., Ndoye, O., Cisse, N., et Sembene, M. (2015). Senna occidentalis $\mathrm{L}$., une plante prometteuse dans la lutte contre Caryedon serratus OI. (Coleoptera, Bruchidae), insecte ravageur des stocks d'arachide au Sénégal. Int. J. Biol. Chem. Sci. 9, 1399.

Torres, A.M., Barros, G.G., Palacios, S.A., Chulze, S.N., et Battilani, P. (2014). Review on pre- and post- harvest management of peanuts to minimize aflatoxin contamination. Food Res. Int. 62, 1119.

Udoh, J.M., Cardwell, K.F., et Ikotun, T. (2000). Storage structures and aflatoxin content of maize in five agroecological zones of Nigeria. J. Stored Prod. Res. 36, 187-201. 